\title{
State of emergency medicine in South Africa
}

\author{
Lee A. Wallis • Sharadh R. Garach • \\ Annemarie Kropman
}

Received: 7 April 2008 / Accepted: 2 May 2008 / Published online: 17 June 2008

(C) Springer-Verlag London Ltd 2008

\begin{abstract}
Introduction Emergency medicine is a new speciality in South Africa. It was first registered in 2003, and there are now 30 specialists in the country, with 10 new graduates from local registrar training programmes and over 40 trainees on four programmes across the country.

Conclusion Emergency medicine is currently enjoying a governmental focus as part of the preparations towards the FIFA 2010 soccer World Cup. This article discusses the current structure of emergency care in South Africa.
\end{abstract}

Keywords South Africa · Emergency medicine .

Current status

\section{Introduction}

South Africa is a young democracy. After decades of forced segregation, in 1994 the country abandoned the rule of apartheid and entered a new phase. The country consists of nine provinces spread over an area of 1.2 million square kilometres - twice the size of Texas, and around the same size as India (Fig. 1) [1]. The 2007 census revealed a population of 47.9 million, with $11 \%$ living on below one US dollar a day [2]. High levels of poverty and unemployment contribute to an ever increasing burden of disease. South Africa faces a quadruple disease burden: violence,

The views expressed in this paper are those of the authors, and not those of the editors, editorial board or publisher.

L. A. Wallis $(\bowtie) \cdot$ S. R. Garach · A. Kropman

University of Cape Town, Stellenbosch University,

Private Bag X24, Bellville,

7535 Cape Town, South Africa

e-mail: lewallis@pgwc.gov.za
HIV/AIDS, infectious diseases and chronic diseases of lifestyle all take their toll, reducing the average life expectancy to 49 years for males and 52 for females [3]. The incidence of HIV infection is estimated to be $11 \%(5.3$ million people); the infant mortality rate is estimated at 45.2 per 1,000 [3]. Approximately one third of admissions to emergency centres (EC) in South Africa are due to injuries; in comparison, trauma makes up $12 \%$ of admissions in the US and about $8 \%$ in the UK [4].

\section{Health care provision}

Health care is provided in two distinctly different arenas: private and public. The private system caters to less than $20 \%$ of the population yet takes $70 \%$ of finances and resources. South Africa's private health care system provides first-world medicine in comfortable surroundings, although it is not immune to the global shortage of nurses and allied health staff [5]. The public sector serves the rest of the population, with minimal resources. The population served by this sector is predominantly indigent, poor, and uneducated.

The public health system is provided in a tiered structure, with $90 \%$ of interactions occurring in the primary care level in nurse-led clinics, or day hospitals. Most day hospitals do not provide overnight beds but do have a functional EC. They have limited access to laboratory and radiological investigations. Hospital care is provided in three tiers, varying from province to province. District hospitals are the mainstay of care and are staffed by nonspecialist medical officers who cover all disciplines, including emergency care in the EC. Basic investigations are available. Each district hospital refers to a regional hospital where general specialists are based (surgeons, physicians, paediatricians and obstetricians). A higher level 


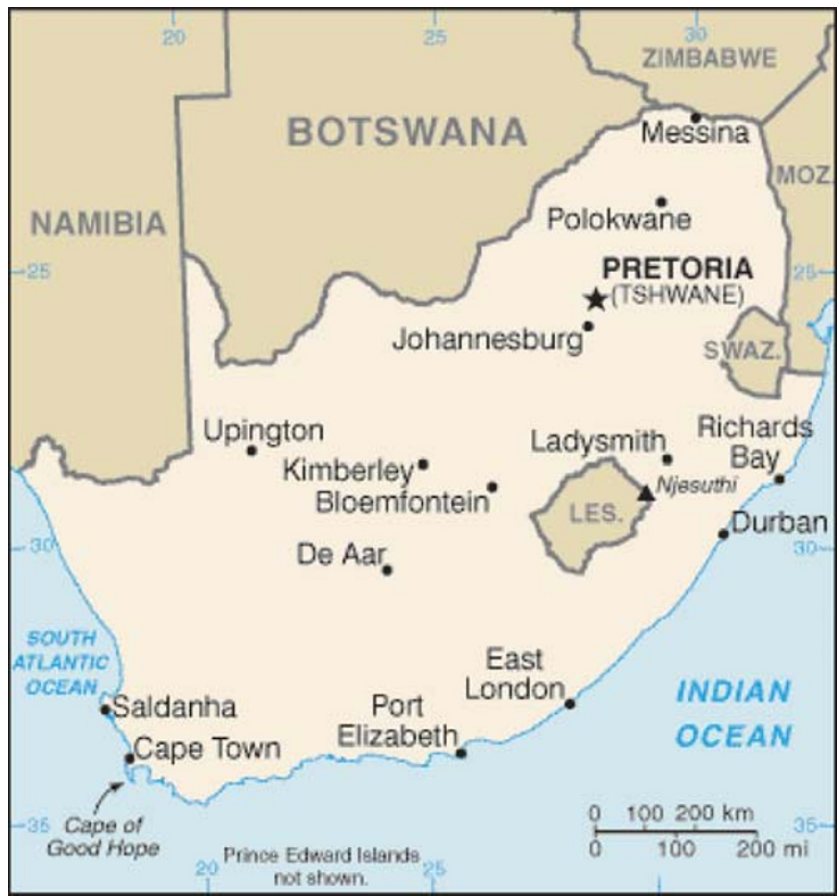

Fig. 1 Map of South Africa

of investigative support is provided. Central hospitals are the pinnacle of the referral system, with less than $2 \%$ of the population receiving care there. They are staffed with all levels of specialists and subspecialists. Emergency care is typically divided into trauma units (staffed by trauma surgeons) and medical emergency units, although this position is slowly changing.

\section{Emergency care}

Emergency care is viewed as a basic human right, enshrined in the constitution of South Africa [6]. However, there is no clarity as to the exact meaning of this right: whether all persons only have the right to basic EC care, or if this includes definitive and intensive care treatment is still hotly debated. All emergency care in the public sector falls under the directorate of Emergency Medical Services (EMS) at the National Department of Health in Pretoria, with policy and guidance being provided to provincial structures.

\section{EMS-based}

Emergency care in the pre-hospital sector also has public and private arenas, with nationally based private services, and provincial public services. These services provide emergency care through basic, intermediate and advanced life support practitioners. Basic life support practitioners have limited life support skills; these skills are enhanced in the intermediate practitioner, while South African para- medics have a wealth of clinical experience and can undertake a higher level of clinical procedures than their peers in many other countries. A review is currently underway to facilitate the collapse of this structure to a two-provider service, with emergency care technicians (trained over 2 years) and emergency care practitioners (paramedics) trained with a 4-year degree programme.

Like hospital services, public EMS services are generally underresourced, understaffed and poorly equipped to service the large areas which they cover. They also suffer from the ongoing global "brain drain" to first world countries. Transport to ECs is mainly road-based, over large distances, with only a few provinces able to utilise rotor wing or fixed wing resources, although the provision of these services continues to increase annually.

Hospital-based

Emergency centres have historically been staffed and run by medical officers and junior doctors, with either surgical cover or often without any senior supervision. There have been no nationally accepted patient management guidelines. Recent moves, however, have emphasised the need for improved emergency care; one of the main drivers behind this was the awarding of the 2010 FIFA soccer World Cup to South Africa. In all parts of the country, ECs are seeing an ever increasing patient load, with above $10 \%$ annual increases in patient volumes [7].

As part of the increased emphasis on emergency care, hospital ECs have seen increased investment and training of staff, although there is still a long way to go in much of the country. Part of the drive behind this process has related to the development of emergency medicine as a speciality.

\section{Emergency medicine speciality development}

Emergency medicine has long been practised in many forms and to a high standard across South Africa without any formal recognition, and indeed elective periods in South Africa have long been seen as a valuable addition to any overseas trainee's curriculum vitae. The recent development of the speciality of emergency medicine was, as in most other countries, driven by a small handful of key individuals.

\section{EMSSA}

The first formal emergency medicine structures were developed in the late 1990s, driven in part by the success of the Trauma Society of South Africa: interested and motivated individuals formed the Emergency Medicine Society of South Africa (EMSSA), which applied for and received affiliate membership in the International Federa- 
tion for Emergency Medicine (IFEM) in 2001. In 2004, full member status was granted.

October 2007 saw Emergency Medicine in the Developing World, a 4-day conference held in Cape Town, with 650 delegates from 42 countries. A second conference is planned for November 2009.

\section{College of emergency medicine}

In 2003, emergency medicine was added to the list of recognised specialities in South Africa. To help the establishment of a respectable speciality with a solid base from which to train registrars, a number of senior emergency medicine practitioners were added to the speciality register under the "grandfather" clause.

Concurrent with this development was the establishment of the College of Emergency Medicine (CEM (SA)). Emergency medicine exams have been held through the South African Colleges of Medicine since 1986: the Diploma in Primary Emergency Care (DipPEC) was initially offered by the College of Family Practitioners, but transferred to the newly formed College of Emergency Medicine in 2004. Applicants must have at least 6 months emergency medicine experience; the exam is intended for those working in emergency care in remote areas. There are currently over 250 diplomates who receive reciprocity with the Fellowship in Immediate Medical Care of the Royal College of Surgeons in Edinburgh.

The main role of the College is examination of trainees in the speciality, and to this end biannual fellowship examinations are held. The primary tests basic sciences (anatomy, pharmacology, physiology and pathology) and may be taken by any fully qualified South African doctor. The final fellowship (FCEM) examination may be taken after 3 years of speciality registrar training, and tests clinical emergency medicine in written and clinical sections. The first FCEM by examination was awarded in late 2007.

\section{University training programmes}

The Division of Emergency Medicine at the University of Cape Town (UCT) was established in 2001, and initially offered a Master of Philosophy (MPhil) degree by examination and dissertation. After the registration of the speciality, UCT commenced a Master of Medicine in Emergency Medicine (MMed) programme in 2004. This programme is now shared with Stellenbosch University, and has 32 trainees; similar programmes are offered at the University of the Witwaterstrand (eight trainees), Pretoria University (four) and Limpopo University (two). Plans are in place to expand the training programmes and increase the number of universities offering such training.

The MMed rotation involves 4 years of 3-month blocks, and includes EMS, intensive care, obstetrics and gynaecology, internal medicine, surgery, and paediatrics. The rest of the training time is spent in ECs. The first 10 registrars graduated from UCT in late 2007 and are now taking up specialist posts.

\section{The future}

The development of the speciality continues apace, and increasing numbers of emergency physicians are now present on the floor of emergency centres across the country. The benefits of such staffing are clear, and are seen in South Africa as they are in other countries where emergency physicians are working. However, resource limitations will continue and for the foreseeable future, demand will far outstrip supply of adequately trained emergency care practitioners.

Key areas to address as emergency medicine moves forward include increased advocacy at a provincial and national level; shop floor training of junior medical staff (and non-medical personnel); outreach programmes to rural, district and day hospitals; continuing medical education programmes, and establishment of local protocols and guidelines. The 2010 FIFA soccer World Cup will be a key test of our development.

Acknowledgements The authors wish to thank the tireless efforts of those personnel who continue to provide excellent emergency care in difficult and trying circumstances throughout South Africa.

Conflicts of interest The authors have no conflicts of interest or disclosures.

\section{References}

1. Central Intelligence Agency, available from https://www.cia.gov/ library/publications/the-world-factbook/print/sf.html accessed 6 April 2008

2. Midyear population estimates-2007. StatsSA, Pretoria. 2007

3. Unicef, available from www.unicef.org/inforbycountry/southafrica statitics.html accessed 6 April 2008

4. Balfour C (2002) Training for trauma. SAMJ 92(10):790-791

5. American Association of Colleges of Nursing http://www.aacn.nche. edu/Media/FactSheets/NursingShortage.htm accessed 6 April 2008

6. South African Government, available from http://www.info.gov.za/ documents/constitution/1996/a108-96.pdf accessed 6 April 2008

7. Wallis LA, Twomey M (2007) Workload and casemix in Cape Town emergency departments. S Afr Med J 97:1276-1280

Prof. Lee A. Wallis received his $\mathrm{MBChB}$ from the University of Edinburgh in 1993. He completed his emergency medicine training with the Royal Navy in the UK, before relocating to Cape Town in 2002. He is currently professor and head of the Division of Emergency Medicine, University of Cape Town. His research interests are in the field of emergency triage. He was awarded his MD in 2005 for his work on paediatric triage in mass casualty situations. He is the president of the Emergency Medicine Society of South Africa (EMSSA). 